\title{
Hypercalcaemic Crisis Due to Primary Hyperparathyroidism: Report of Two Cases
}

\author{
Tatiana Daniela Sala1,2, Simona Mureşan ${ }^{3}$, Ramona Roman ${ }^{1}$, Alexandra Lazăr1,4, Răzvan \\ Ion², Ionela Paşcanu1,5 \\ 1 University of Medicine, Pharmacy, Sciences and Technology of Târgu Mureş, Romania \\ 2 Emergency Mures County Hospital, Surgery Clinic no.2, Târgu Mureş, Romania \\ 3 Physiology Department, University of Medicine, Pharmacy, Sciences and Technology of Târgu Mureş, Romania \\ ${ }^{4}$ Anesthesiology and Intensive Care Clinic no. 1, Târgu Mureş, Romania \\ ${ }^{5}$ Endocrinology Clinic, Târgu Mureş, Romania
}

\begin{abstract}
Introduction: A hypercalcaemic crisis, also called para thyrotoxicosis, hyper parathyroid crisis or parathyroid storm, is a complication of primary hyperparathyroidism (PHPT) and an endocrinology emergency that can have dramatic or even fatal consequences if it is not recognised and treated in time.
\end{abstract}

Case presentation: Two cases presented in the emergency department with critical hypercalcaemic symptoms and severe elevation of serum calcium and parathyroid hormone levels, consistent with a hypercalcaemic crisis. The first case, a 16-year-old female patient, had imaging data that highlighted a single right inferior parathyroid adenoma and a targeted surgical approach was used. The second case, a 35-year-old man was admitted for abdominal pain, poor appetite, nausea and vomiting. Laboratory tests revealed severe hypercalcaemia, hypophosphatemia and an increased serum iPth level. There was no correlation between scintigraphy and ultrasonography, and a bilateral exploration of the neck was preferred, resulting in the exposure of two parathyroid adenomas. The patients were referred for surgery and recovery in both cases was uneventful

Conclusion: These cases support the evidence that surgery remains the best approach for patients with a hypercalcaemic crisis of hyperparathyroidism origin, ensuring the rapid improvement of both the symptomatology and biochemical alterations of this critical disease.

Keywords: primary hyperparathyroidism, hypercalcaemic crisis, parathyroid crisis, endocrinology emergency, surgery Received: 18 December 2018 / Accepted: 22 January 2019

\section{INTRODUCTION}

Primary hyperparathyroidism (PHPT) is ranked second in frequency amongst endocrine diseases, being caused in more than $80 \%$ of cases by a solitary parathyroid adenoma. Other, less frequent causes are multiple parathyroid hyperplasia, double adenomas and parathyroid cancer [1]. A hypercalcaemic crisis, also called para thyrotoxicosis, hyper parathyroid crisis or parathyroid storm, is a complication of PHPT and an endocrinology emergency that can have dramatic or even fatal consequences if it is not recognised and treated in time expeditiously [2,3]. A diagnosis is established in the biochemical context of hyperparathyroidism when there is an elevated serum intact parathyroid hormone level (iPth) together with a substantial increase in the serum calcium level more than $14 \mathrm{mg} / \mathrm{dL}$, associated with an acute onset of symptoms.

Symptoms include dehydration, anorexia and vomiting, mental alteration, cardiac arrhythmia, impaired cardiac and renal function and finally death $[3,4]$, and the severity of the of symptoms tends to be proportional to the level of hypercalcaemia. In such cases, emergency hospitalisation and the rapid establishment of intensive care measures to restore calcium levels to within normal limits is mandatory. In most instances, 
surgical treatment, consisting of the excision of the parathyroid adenoma, is required.

Two cases which presented in the emergency department of Emergency County Hospital Targu Mures, Romania with hypercalcaemic symptoms and a severe elevation of serum calcium and parathyroid hormone levels, consistent with a hypercalcaemic crisis of hyperparathyroidism origin are reported.

\section{CASE 1}

A 16-year-old female patient, body mass index (BMI) of $24 \mathrm{~kg} / \mathrm{m} 2$, was admitted to the hospital's endocrinology department, complaining of nausea, vomiting, dehydration and slight alteration of mental status, having experienced confusion over the three preceding days. Biochemical tests showed severe hypercalcaemia a serum calcium level of $14.9 \mathrm{mg} / \mathrm{dL}$, [normal value $(\mathrm{NV})$ 9-11 mg/dL], a serum phosphorus level of $1.8 \mathrm{mg} / \mathrm{dL}$ [NV:2.7-4.5 mg/dL] ) and a serum iPth level of $184 \mathrm{pg} /$ $\mathrm{mL}$ [NV:20-65 pg/mL]. The patient was known to have had repeated admissions for bilateral renal lithiasis, including a left ureterolithotomy for an obstructive calculus and secondary hydronephrosis. Her medical records showed a steadily elevated value of iPth and serum calcium in the period preceding hospital admission. On admission, initial treatment consisted of oral and parenteral hydration, 3-3.5 L/day, and furosemide
(Zentiva SA, Bucharest, Romania), 10 mg, 2-3 times/ day, disodium pamidronate (Dr Reddy Laboratories Ltd. India), intravenously (IV) $30 \mathrm{mg}$ /day was also given to reduce serum calcium levels. In the days following the emergency hospitalisation, calcium levels fell but constantly remained above the normal upper limit of $12.89 \mathrm{mg} / \mathrm{dL}$.

A cervical ultrasound showed a round-oval hypoechoic mass, $0.54 \times 0.77 \times 1.54$, on the lower part of the right thyroid lobe, clearly delimited and of a moderate colour, according to the Doppler vascular signal. Parathyroid scintigraphy with Tc 99m-Sestamibi highlighted an increased capture of the radiotracer on early and late acquisition, at one hour and 30 minutes postinjection. A mass located on the lower part of the right inferior thyroid lobe was detected, indicative of a parathyroid adenoma (Figure 1 and 2).

The patient was referred for emergency surgical treatment and considering the imaging data that highlighted a parathyroid adenoma below the right inferior thyroid lobe, open minimally invasive parathyroidectomy (OMIP) was carried out. Intraoperatively, a friable reddish-brown tumour of about $1.5 \mathrm{x} 1 \mathrm{~cm}$ was excised below the right thyroid lobe, and the histopathological report confirmed parathyroid tissue hyperplasia. (Figure 3).

Postoperatively, serum calcium levels were strictly monitored and high doses of IV calcium, 6-8 g/day,

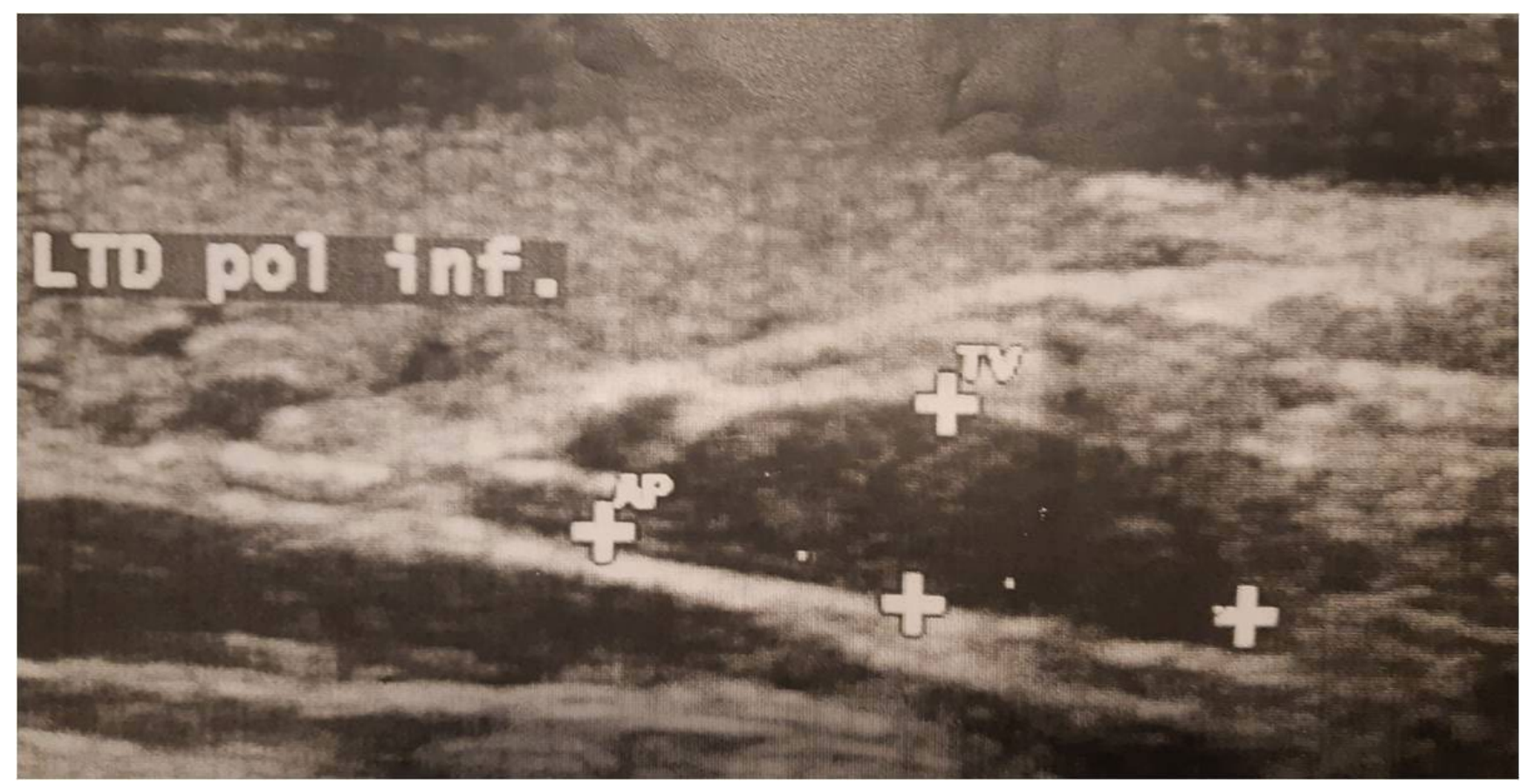

Fig. 1. Cervical ultrasonography indicating an hypoechogenic mass, corresponding to the right inferior parathyroid adenoma. 

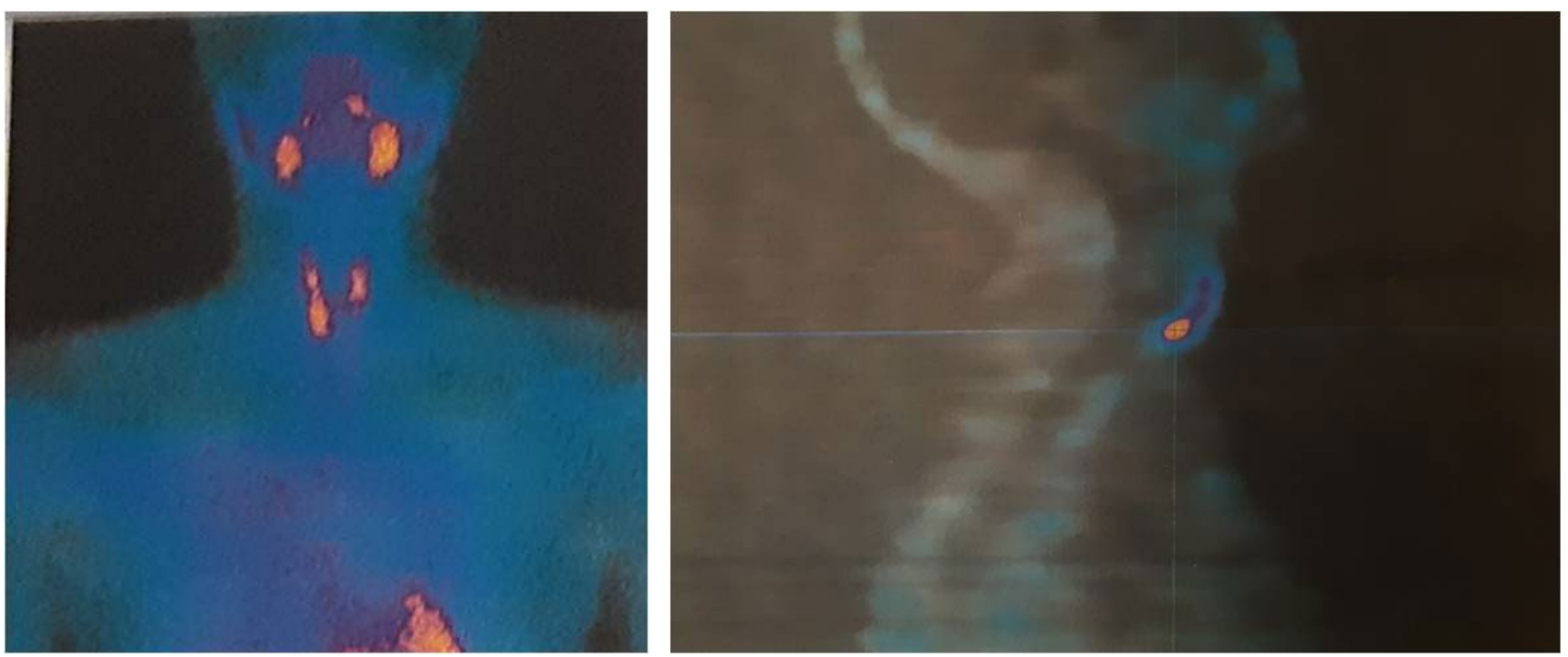

Fig. 2. Parathyroid scintigraphy with Tc $99 \mathrm{~m}$-sestamibi showing uptake in the right inferior parathyroid gland

was given to keep the calcium value within the normal range.

The patient's recovery was uneventful, and she was discharged three days postoperatively. Oral calcium supplements, $2-4 \mathrm{~g} /$ day, and vitamin $\mathrm{D}, 0.5-1 \mu \mathrm{g} / \mathrm{day}$, to be taken for one month after the operation, were prescribed. The patient's condition continued to improve in the following period. The serum calcium level reached $9.6 \mathrm{mg} / \mathrm{dL}$ and the parathyroid hormone level $31.5 \mathrm{pg} /$ $\mathrm{mL}$ after one month. At the three month follow-up, the patient was asymptomatic, with a total serum calcium level of $9.5 \mathrm{mg} / \mathrm{dL}$ and a normal parathyroid hormone level of $21.2 \mathrm{pg} / \mathrm{mL}$, these values being achieved without medication.

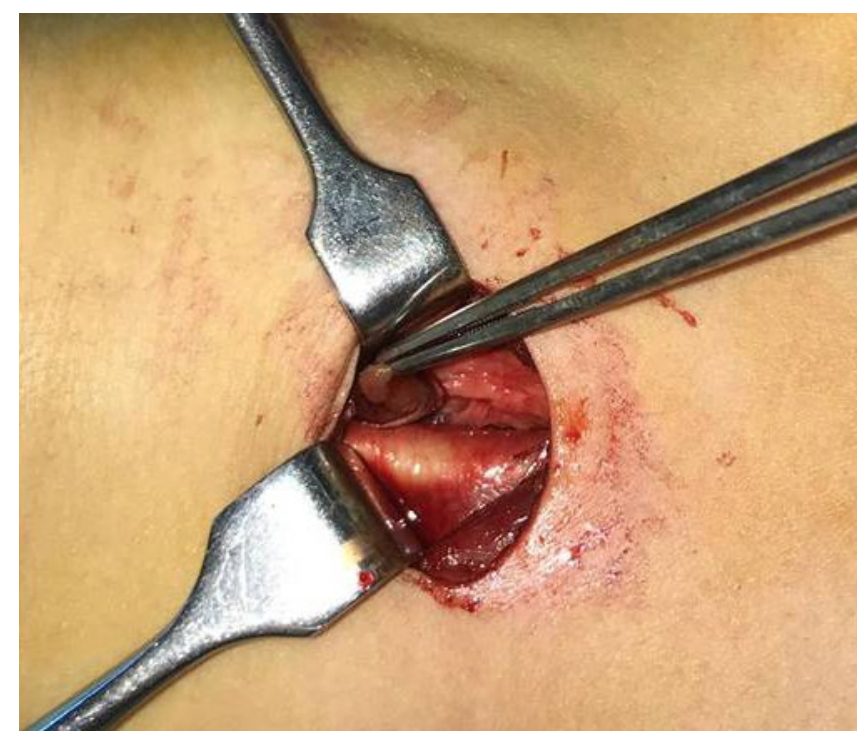

Fig. 3. Right open minimally invasive parathyroidectomy (OMIP)- the right inferior parathyroid adenoma.
口CASE 2

A 35-year-old man, BMI of $29 \mathrm{~kg} / \mathrm{m} 2$, was admitted to the emergency department of Emergency Mures County Hospital, complaining of abdominal pain, poor appetite, nausea, vomiting and generalised weakness that had started two weeks earlier. The patient had a history of a perforated gastric ulcer, ten years earlier, and several episodes of bilateral renal lithiasis.

Upon admission, initial tests excluded an acute abdomen. However, the abdominal pain was refractory to symptomatic treatment. Laboratory tests revealed a serum calcium level of $15.2 \mathrm{mg} / \mathrm{dL}$, a serum phosphate level of $2.6 \mathrm{mg} / \mathrm{dL}$ and a serum potassium level of $2.9 \mathrm{mmol} / \mathrm{L}$, indicative of severe hypercalcaemia, hypophosphatemia and hypopotassaemia respectively.

A hyperparathyroid crisis and primary hyperparathyroidism were suspected, which were subsequently confirmed by an increased serum iPth level of $295 \mathrm{pg} /$ $\mathrm{mL}$. Initial emergency treatment included aggressive fluid resuscitation of 4-4.5 L/day, furosemide (Zentiva SA, Bucharest, Romania), 10 mg, 2-3 times/day, calcitonin (Novartis Pharma Stein AG., Switzerland) 5 $\mathrm{UI} / \mathrm{kg}$ IV twice a day and disodium pamidronate (Dr Reddy Laboratories Ltd. India) $30 \mathrm{mg} /$ day IV, to reduce calcium levels.

A cervical ultrasound revealed an imprecise hypoechogenic mass of $5 \times 7 \times 6 \mathrm{~mm}$, below the left thyroid lobe. Parathyroid scintigraphy with Tc $99 \mathrm{~m}$-Sestamibi revealed an area of late tardive capture of radiotracer below the right thyroid lobe, compared to no alteration on the left parathyroid area, suggestive of a right 
inferior parathyroid adenoma. Five days after admission the patient was still symptomatic with a serum calcium level of $13.1 \mathrm{mg} / \mathrm{dL}$. He was referred for an urgent parathyroidectomy. Given the lack of targeting, parathyroidectomy was performed through a standard cervicotomy with the bilateral exploration of parathyroid areas. Two parathyroid adenomas were excised, a left inferior adenoma of $1 \times 1 \mathrm{~cm}$ and a right inferior adenoma of $1 \times 0.5 \mathrm{~cm}$. The histopathological report confirmed parathyroid tissue hyperplasia.

Immediately after surgery, the iPth value was recorded at $51.2 \mathrm{pg} / \mathrm{mL}$. However, the patient developed a severe postoperative hypocalcaemia with a serum calcium level of $7.1 \mathrm{mg} / \mathrm{dL}$. This was treated with high doses of IV calcium, 8-10 gr/day, for the next four postoperative days. Normal calcium levels were established, and he was discharged after one week. Oral calcium supplements, 2-4 gr/day and vitamin D, 0.5-1 $\mu \mathrm{gr} /$ day, were prescribed, to be taken for one month postoperatively. The patient was reviewed after three months and then intermittently for three years postoperatively during which time calcium and serum phosphorus values remained within normal limits with the late iPth value of $41.6 \mathrm{pg} / \mathrm{mL}$.

\section{DISCUSSION}

Primary hyperthyroidism is listed alongside malignancy as the primary cause of hypercalcaemia $[1,4,5]$.

A hypercalcaemia crisis does not have a clear definition in the literature, but markedly elevated serum calcium values above $14 \mathrm{mg} / \mathrm{dL}$, accompanied by the signs and symptoms of hypercalcaemia are recognised as relevant diagnostic criteria [2,3]. Also called a parathyroid crisis or parathyrotoxicosis, Hanes first described it in 1939 , has a prevalence of $1-2 \%$ and is recognised as an endocrine emergency, invariably fatal in the absence of optimal treatment [6].

The symptoms of PHPT are heterogeneous, including neuromuscular, digestive, bone and kidney symptoms. The classic indicative aphoristic mnemonic "stones, bones, abdominal moans and groans psychic" is rarely seen these days, especially in western countries, where the disease usually progresses in an oligosymptomatic manner [7]. However, there are geographical areas, especially in less developed countries, where severe manifestations of PHPT are still commonly encountered, and where the disease frequently goes undiagnosed with treatment often commencing at a later than optimum time [8]. This is also the case in Romania, where symptomatic cases, such as those presented, are often encountered. Both patients were diagnosed in a difficult phase of the disease. In Case 1, a 16-year-old teenager, the disease caused bilateral renal lithiasis, the patient having to undergo surgery for this condition. The patient In Case 2, the patient had had a perforated ulcer at 18 years of age, probably because of undiagnosed PHPT. Irregular digestive symptoms persisted, and an episode of acute pancreatitis occurred. The emergency admission to hospital for a hypercalcaemic crisis was also due to continuous abnormal digestive symptoms.

Surprisingly, in Romania, situations are frequently observed where PHPT is diagnosed only when cervical tumours are discovered during routine ultrasound examinations in patients with long-standing heterogeneous symptoms. In such cases, it is not a rare event that large parathyroid adenomas are found intraoperatively [8].

The diagnosis of PHPT and implicitly of a hypercalcaemic crisis frequently depends on the results of biochemical tests. Measurements of the total serum calcium are frequently inaccurate and corrected total serum calcium on albumin, or ionic calcium are more useful in diagnosis. An increased serum level of iPth usually confirms a diagnosis of PHPT.

Imagistics is not a diagnostic criterion in PHPT but helps the surgeon to locate an adenoma or adenomas and to target the appropriate surgical area [9]. In most PHPT cases a single adenoma is being dealt with, and targeting is an advantageous technique which allows minimally-invasive interventions. Case1 represents this well. Scintigraphy with Tc 99m-Sestamibi and a cervical ultrasound confirmed the location of the adenoma and allowed its targeted excision. A meta-analysis of several studies showed that sensitivity of scintigraphy with Tc $99 \mathrm{~m}$-Sestamibi compared to the high-resolution US was $88 \%$ versus $78 \%$ for single adenomas, and the combination of the two methods provides the best sensitivity for targeting the operation site [10]. The sensitivity of these methods decreases significantly in the case of double adenomas and especially in the case of four-gland hyperplasia [10]. The second case is indicative of these views. In Case 2, there was no correlation between the scintigraphy and ultrasonography, and so a bilateral exploration of the neck was opted for, revealing two parathyroid adenomas. Apparently, in this situation, a targeted operation would have been followed by persistent hyperparathyroidism. A solution not yet 
available in the clinic, but which would allow a better targeting, would be intraoperative parathyroid hormone assay. Indeed, it appears that a decrease of more than $50 \%$ over the baseline parathyroid hormone value at 10-15 minutes after resection, is suggestive of a single adenoma. In the absence of such a decrease over baseline parathyroid hormone values, it is more likely that a bilateral mass or four-gland hyperplasia exists and the procedural approach of a minimally invasive operation would be converted to one which allowed for exploration of other additional sites [11].

Even though the mortality rate gradually declined in recent decades due to earlier diagnosis and improved medical treatment, the diagnosis of a hyperparathyroid crisis still demands emergency measures as it is a critical condition with high mortality in the absence of therapeutic intervention [12]. Initial treatment of moderate and severe hypercalcaemia consists of IV hydration and afterwards loop diuretics, carried out with 2-4 L/day of saline solution for two-three days depending on calcaemia, the degree of dehydration and patient's tolerance to rehydration.

A repeated dose of diuretics can be useful after rehydration which will prevent overloading. Patients who do not tolerate rapid IV rehydration, particularly those presenting with renal or cardiac diseases, may require dialysis. In the cases presented, although the values of the calcaemia at admission were high, both patients responded favourably to rehydration and administration of furosemide, $10 \mathrm{mg}, 2-3$ times daily. In recent years, calcitonin and bisphosphonate have been used to treat a parathyroid crisis due to their effect of inhibiting calcium bone resorption, though reports suggest that the results were not as good as expected $[13,14]$. However, it has been suggested that they might be used in moderate and severe hypercalcaemia as a bridge therapy to parathyroidectomy, when rapid rehydration is contraindicated [12].

Rehydration, stimulation of diuresis and daily as well as the inhibition of bone resorption are all emergency therapeutic measures useful in treating the critically ill hypercalcaemic patient, though surgery remains the definitive and pathogenic treatment of a parathyrotoxic crisis [6]. As shown in both the reported cases, parathyroidectomy succeeds by rapidly and substantially reducing the serum iPth level, to break the pathogenic chain in PHPT and HC crisis. IPth serum values rapidly normalised in both cases and serum calcium values decreased gradually over the following few days.
One should be mindful that in such patients there is a risk of postoperative hungry bone syndrome (HBS), its severity depending on preoperative serum calcium levels and bone damage secondary to hyperparathyroidism. HBS is not specific to PHPT, being also found in patients with secondary hyperparathyroidism (SHPT) of renal origin. In these cases, the pathogenic mechanism is more complex, and the treatment has particularities related to chronic dialysis [15]. A rapid reduction in postoperative calcaemia is not desirable in patients with high preoperative serum calcium levels. In contrast to post-thyroidectomy hypocalcaemia, usually transient, subclinical and secondary to incidental parathyroidectomy [16], hypocalcaemia after the resection of a parathyroid adenoma may be severe and responsible for fatal cardiac complications. For this reason, several authors have recommended that IV calcium substitution is initiated immediately postoperatively [17]. In both cases, serum calcium values were continuously monitored, and high doses of IV calcium were administered to keep the calcium levels within normal limits. Finally, both patients were discharged with normal calcium levels with oral calcium and vitamin D substitution.

The development of imaging techniques has enabled parathyroidectomy to be targeted. Different RCTs have demonstrated the benefits of a minimally invasive approach which result in reduced postoperative pain, shorter operative time, earlier discharge and better cosmetic results [18].

A minimally invasive approach was preferred this in Case 1. In any other situation, when the diagnosis of localisation is not certain, or there is suspicion of double or multiple masses, the only surgical solution remains bilateral cervical exploration with subtotal excision of the parathyroid hyperplasic parenchyma and maintenance of auto implantation in the cervical or distal region. This is illustrated by Case 2 , where a discordance between the two imagistic investigations was noted. Indeed, intraoperative bilateral exploration revealed double diseased sites and allowed the excision of both parathyroid adenomas. Symptom resolution, normalisation of calcium levels, and maintaining iPth within normal limits during follow-up confirmed a successful outcome for both patients.

\section{CONCLUSION}

Primary hyperparathyroidism and its hypercalcaemic complication are frequently misdiagnosed due 
Available online at: www.jccm.ro

to atypical symptomatology. Emergency conservative treatment, including aggressive fluid resuscitation, diuretics, calcitonin and bisphosphonates may help to stabilise the critically ill patient providing time for further imagistic investigations. The current reports support the evidence that surgery remains the best approach for patients with a hypercalcaemic crisis of hyperparathyroidism origin, ensuring the rapid improvement of both the symptomatology and biochemical alterations of this disease.

\section{CONFLICT OF INTEREST}

None to declare.

\section{REFERENCES}

1. Marcocci C, Cetani F. Clinical practice. Primary hyperparathyroidism. N Engl J Med. 2011;365:2389-97.

2. Singh DN, Gupta SK, Kumari N, et al. Primary hyperparathyroidism presenting as hypercalcemic crisis: twenty-year experience. Indian J Endocrinol Metab. 2015;19:100-105.

3. Cannon J, Lew JI, Solorzano CC. Parathyroidectomy for hypercalcemic crisis: 40 years' experience and long-term outcomes. Surgery. 2010;148:807-12.

4. Walsh J, Gitoes N, Selby P. Society of Endocrinology Clinical Committee, Society for Endocrinology Endocrine Emergency Guidance. Emergency management of acute hypercalcemia in adult patients. Endocr Connect 2016;5:G9-G11.

5. lacob A, Zazgyva A, Ormenisan A, Mezei T, Sin A, Tilinca $M$. Effectiveness of fine-needle aspiration cytology in the diagnosis of lateral cervical non-thyroid tumors. Medicine (Baltimore), 2016;95:e4448.

6. Sherwood JK, Garcia M, Ackroyd FW, et al. Hyperparathyroid crisis reviewed: a role for parenteral cimetidine. Am Surg1986;52:320-32.

7. Silveberg SJ, Bilezikian JP. The diagnosis and management of asymptomatic primary hyperparathyroidism. Nat Clin Pract
The Journal of Critical Care Medicine 2019;(5)1 • 39

Endocrinol Metab. 2006;2:494-503.

8. Neagoe RM, Sala DT, Borda A, Mogoantă CA, Muhlfay G. Clinicopathologic and therapeutic aspects of giant parathyroid adenomas- three case reports and short review of the literature. Rom J Morphol Embriol. 2014;55:669-74.

9. Beck W, Lew JI, Solorzano CC. Hypercalcemic crisis in the era of targeted parathyroidectomy. J Surg Res. 2011;171:404-408.

10. Lumachi F, Zucchetta P, Marzola MC, et al. Advantages of combined technetium-99m-sestamibi scintigraphy and high-resolution ultrasonography in parathyroid localization: Comparative study in 91 patients with primary hyperparathyroidism. Eur J Endocrinol. 2000;143:755-60.

11. Suliburk JW, Perrier ND. Primary hyperparathyroidism. Oncologist. 2007;12:644-53.

12. Phitayakorn R, McHenry CR. Hyperparathyroid crisis: use of bisphosphonates as a bridge to parathyroidectomy. J Am Coll Surg. 2008;206:1106-15.

13. Udelsman R. Six hundred and fifty-six consecutive explorations for primary hyperparathyroidism. Ann Surg. 2002;235:66570.

14. Khan AA, Hanley DA, Rizzoli R, et al. Primary hyperparathyroidism: review and recommendations on evaluation, diagnosis, and management. A Canadian and international consensus. Osteoporos Int. 2017;28:1-19.

15. Neagoe RM, Mureșan M, Voidăzan S, Pașcanu I, Radu CP, Sala DT. Subtotal parathyroidectomy versus total parathyroidectomy with autotransplant in secondary hyperparathyroidism - a single-centre prospective cohort of 43 patients. Endokrinologia Polska 2016;67:202-209.

16. Neagoe RM, Cvasciuc IT, Muresan M, Sala DT. Incidental parathyroidectomy during thyroid surgery- risk, prevention and controversies; an review. Acta Endo (Buc) 2017;13:46775.

17. Ameerudden S, He X. Management and surgical treatment of parathyroid crisis secondary to parathyroid tumors: report of four cases. Int Med Case Rep J. 2011;15:59-66.

18. Russel CF, Dolan SJ, Laird JD. Randomised clinical trial comparing scan-directing unilateral versus bilateral exploration for parathyroidectomy due to solitary adenoma. Br J Surg 2006;93:418-21. 